\section{Alterations in chromosomal synapses and DNA repair in apoptotic spermatocytes of Mus m. domesticus}

\author{
E. Ayarza, ${ }^{1,2}$ M. González, ${ }^{1}$ F. López, ${ }^{1}$ \\ R. Fernández-Donoso, ' J. Page, ${ }^{3}$ \\ S. Berrios ${ }^{1}$
}

'Programa Genética Humana, ICBM, Facultad de Medicina, Universidad de Chile, Santiago, Chile

2Departamento Tecnología Médica, Facultad de Medicina, Universidad de Chile, Santiago, Chile

${ }^{3}$ Departamento de Biología Celular, Universidad Autónoma de Madrid, Spain

\section{Abstract}

We investigated whether apoptotic spermatocytes from the mouse Mus $m$. domesticus presented alterations in chromosomal synapses and DNA repair. To enrich for apoptotic spermatocytes, the scrotum's temperature was raised by partially exposing animals for $15 \mathrm{~min}$ to a $42^{\circ} \mathrm{C}$ water bath. Spermatocytes in initial apoptosis were identified in situ by detecting activated caspase-9. SYCP1 and SYCP3 were markers for evaluating synapses or the structure of synaptonemal complexes and Rad51 and $\gamma \mathrm{H} 2 \mathrm{AX}$ for detecting DNA repair and chromatin remodeling. Apoptotic spermatocytes were concentrated in spermatogenic cycle stages III-IV (50.3\%), XI-XII (44.1\%) and IX-X (4.2\%). Among apoptotic spermatocytes, $48 \%$ were in middle pachytene, $44 \%$ in metaphase and $6 \%$ in diplotene. Moreover, apoptotic spermatocytes showed several structural anomalies in autosomal bivalents, including splitting of chromosomal axes and partial asynapses between homologous chromosomes. $\gamma \mathrm{H} 2 \mathrm{AX}$ and Rad51 were atypically distributed during pachytene and as late as diplotene and associated with asynaptic chromatin, single chromosome axes or discontinuous chromosome axes. Among apoptotic spermatocytes at pachytene, $70 \%$ showed changes in the structure of synapses, $67 \%$ showed changes in $\gamma \mathrm{H} 2 \mathrm{AX}$ and Rad51 distribution and 50\% shared alterations in both synapses and DNA repair.

Our results showed that apoptotic spermatocytes from Mus m. domesticus contain a high frequency of alterations in chromosomal synapses and in the recruitment and distribution of DNA repair proteins. Together, these observations suggest that these alterations may have been detected by meiotic checkpoints triggering apoptosis.

\section{Introduction}

In sexually mature males, Sertoli cells recognize and quickly remove apoptotic cells by phagocytosis. ${ }^{1}$ During the spermatogenetic cycle, about $0.2 \%$ of cells may be undergoing apoptosis. This is a relative percentage value, representing the number of apoptotic germ cells for every 100 Sertoli cells. $^{2}$ Apoptotic germ cells are mainly dividing spermatogonia and spermatocytes in meiotic prophase. The activities of caspases- $3,-8$ and -9 are similar in apoptotic germ and somatic cells, suggesting that these cell lineages share apoptotic pathways. ${ }^{3}$ During the first meiotic prophase, chromosomal recognition, pairing, synapses and recombination between homologous chromosomes are closely interrelated and sequentially occurring processes. ${ }^{4,5}$ There are also structural changes in chromosomes associated with these processes such as, the formation of synaptonemal complexes (SCs) and the recruitment of specific proteins, including DNA repair proteins. The SC is a proteinaceous structure assembled between the two homologues that consists of two lateral elements, one per homologue, bound in a zipperlike manner, by the central element. The chromatin, organized in loops, is connected to the SC's lateral elements. ${ }^{6-8}$ Several proteins are associated with the SC. SYCP3 protein is the main constituent of the lateral elements or axes of each pair of homologous chromosomes, while SYCP1 protein is the main constituent of the central element. ${ }^{8,9}$ The Spo11 protein initiates meiotic recombination by generating DNA double-strand breaks (DSBs) which are also crucial to the successful recognition/ pairing between homologous chromosomes. ${ }^{10,11}$ DSBs induce local chromatin remodeling, mainly by introducing specific histone modifications such as phosphorylation of histone $\mathrm{H} 2 \mathrm{AX}(\gamma \mathrm{H} 2 \mathrm{AX})$, which in turn promotes the recruitment of the DNA repair proteins. ${ }^{12}$ As DSBs are repaired and chromosomes synapsed there is a decrease in $\gamma \mathrm{H} 2 \mathrm{AX} .{ }^{13}$ Thus, at pachytene, $\gamma \mathrm{H} 2 \mathrm{AX}$ remains associated only with the sex chromosomes' unsynapsed chromatin..$^{14,15}$ Recombination and synapsing between homologous chromosomes occurs concurrently with the recruitment of the DNA repair/recombinases $\operatorname{Rad} 51$ and Dmc1. ${ }^{16,17} \operatorname{Rad} 51$ and Dmc1 have been shown to form numerous foci named early recombination nodules. Early recombination nodules participate in DSBs, early DNA repair and recombination. ${ }^{11,18}$ During the mid pachytene, a reduced number of late recombination nodules indicate the effective crossing-over events between homologous chromosomes. ${ }^{18}$ Desphosphorylation of histone $\gamma \mathrm{H} 2 \mathrm{AX}$ in synapsed chromatin occurs concurrently with
Correspondence: Soledad Berrios, Programa Genética Humana, ICBM, Facultad de Medicina, Universidad de Chile, 1027 Independencia Ave., Santiago 8380453, Chile.

Tel. +56.2.29786462.

E-mail: sberrios@med.uchile.cl

Key words: Apoptosis; chromosome synapsis and recombination; heat stress; meiosis; Mus $\mathrm{m}$. domesticus; spermatocytes.

Contributions: SB, experiments concept and design, paper writing; EA, MG, FL, experiments performing; SB, EA, JP, RFD, data analysis.

Conflict of interest: the authors report no conflicts of interest.

Funding: This work was supported by FONDECYT Project \#1120160 and VID Universidad de Chile, and Project CGL2014-53106-P from Ministerio de Economía y Competitividad (Spain).

Acknowledgments: we wish to thank M. Berrios for helpful suggestions to the manuscript.

Received for publication: 3 May 2016. Accepted for publication: 1 June 2016.

This work is licensed under a Creative Commons Attribution-NonCommercial 4.0 International License (CC BY-NC 4.0).

(O)Copyright E. Ayarza et al., 2016

Licensee PAGEPress, Italy

European Journal of Histochemistry 2016; 60:2677 doi:10.4081/ejh.2016.2677

the disappearance of Rad51 and Dmc1 from recombination nodules. ${ }^{11,19}$ It has been reported that exposing adult male mice to increasing temperature (temperature stress) resulted in a loss of germ cells by apoptosis as well as poor structural quality of spermatozoa. Pachytene spermatocytes and spermatids are the germ cells most susceptible to higher temperature exposure..$^{20-22}$ To our knowledge, there is little information associating apoptotic spermatocytes with anomalies in chromosomal synapses and/or DNA repair during meiosis. Exposing adult male mice to a brief and moderate rise in temperature may provide an excellent experimental model for the enrichment of spermatocytes undergoing apoptosis thus, facilitating studies directed at determining which chromosomal and DNA repair alterations may be present in these spermatocytes. Using sexually mature male mice in combination with the above temperature raise experimental model we investigated whether apoptotic spermatocytes also contain alterations in the distribution of proteins constituting SCs between homologous chromosomes and/or in the recruitment and distribution of DNA repair proteins. 
Our results showed that alterations in SCs and DNA repair proteins are found more frequently in apoptotic than in normal spermatocytes at prophase I, suggesting that these alterations may be detected by meiotic checkpoints triggering programmed cell death.

\section{Materials and Methods}

Six sexually mature 9 to 12 weeks old male mice (Mus musculus domesticus, $\mathrm{C} 3 \mathrm{H}$ strain) were studied. Mice were fed ad libitum while maintained at $22^{\circ} \mathrm{C}$ on a $12 / 12 \mathrm{~h}$ light/dark cycle. Experimental procedures involving mice were approved by the Medical School's Ethics Review Committee ( ${ }^{\circ}$ CBA \#0441) and by the Chilean National Science Foundation FONDECYTCONICYT's Ethics Review Committee. Care and handling of laboratory animals followed institutional and national guidelines (protocol CBA \#0441 FMUCH).

\section{Induction of apoptosis in seminifer- ous epithelium}

Mice were partially immersed for $15 \mathrm{~min}$ in a thermo-regulated circulating bath kept at $42^{\circ} \mathrm{C}$. After 4 or $5 \mathrm{~h}$ post water immersion, mice were sacrificed as previously described..$^{20,23}$

\section{Immunochemical detection of apoptotic germ cells in seminiferous epithelium}

Testes were surgically removed and fixed for 5 to $6 \mathrm{~h}$ in freshly prepared Bouin solution (75 $\mathrm{mL}$ saturated solution of picric acid in distilled water, $5 \mathrm{~mL}$ of acetic acid, $20 \mathrm{~mL} 37 \%$ (w/v) formalin). Once dehydrated in ethanol, testes were embedded in Paraplast and $5 \mu \mathrm{m}$ thick serial sections made. Preceding immunocytochemistry protein renaturation and antigenic recovery was achieved by exposing histological sections to double distilled water vapor for $30 \mathrm{~min}$. Before probing with antibodies, sections were incubated for $20 \mathrm{~min}$ at $37^{\circ} \mathrm{C}$ with freshly prepared $4 \%(\mathrm{w} / \mathrm{v})$ fetal bovine serum (FBS) blocking solution. Caspase- 9 was detected using rabbit polyclonal abs 32539 and 2320 (Abcam, Cambridge, UK) showing monospecific specificities for caspase-9 in situ. Histological sections with anti-caspase- 9 primary antibodies at a dilution of 1: 200/ 1:500 respectively, in phosphate buffer saline (PBS) supplemented with $2 \%(\mathrm{w} / \mathrm{v}) \mathrm{FBS}$, were incubated in a humidified chamber for $1 \mathrm{~h}$ at $37^{\circ} \mathrm{C}$ or overnight at $4^{\circ} \mathrm{C}$. After incubation with first specific antibodies, sections were washed several times in $1 \mathrm{X}$ PBS. Sections were incubated with labeled secondary antibodies diluted 1:100 in PBS for $45 \mathrm{~min}$ at $37^{\circ} \mathrm{C}$ in a humidified chamber. Secondary antibodies were: FITC-conjugated goat anti-mouse
IgG (Jackson Laboratory, Bar Harbor, ME, USA), FITC-conjugated or Texas Red-conjugated goat anti-rabbit IgG (Jackson Laboratory). Nuclei were stained with DAPI (4', 6-diamino-2phenylindole) (Calbiochem, Billerica, MA, USA) and coverslips mounted with Vectashield (Vector Laboratories Inc., Burlingame, CA, USA). Alternatively, biotinylated goat anti-rabbit IgG were used in combination with Kit BIOCARE'S 4plus ${ }^{\mathrm{TM}}$ Detection Systems (immunoperoxidase and peroxidase and diaminobenzidine Streptoavidin-peroxidase and diaminobencidine) and according to the manufacturer's instructions. These sections were treated for 5 min with $5 \%(\mathrm{w} / \mathrm{v})$ periodic acid, following by 40 min incubation in Schiff's reagent. Nuclei were stained with Mayer's hematoxylin essentially as described by McManus. ${ }^{24}$ The different stages of the cycle of the seminiferous epithelium were defined according the mouse spermiogenesis described by 0akberg. ${ }^{25}$ The apoptotic condition of a specific state in seminiferous epithelia was determined when in a given cross section of seminiferous tubules at such stage at least three or more spermatocytes show the presence of caspase-activated positive reaction. ${ }^{20,26}$ At least 10 histochemical observations for each state of the seminiferous epithelium were recorded.

\section{In situ detection of apoptosis in iso- lated germ cells}

We used a squashing technique that preserves relatively well the nuclear volume and cytoplasm of germ cells and was performed essentially according to Page. ${ }^{27}$ Once seminiferous tubules were fixed in $1 \%(\mathrm{w} / \mathrm{v})$ buffered formalin, they were carefully pressed so that cells were allowed to exit the tubules and move onto the surface of clean microscope slides. Coverslips were used to gently apply pressure in order to separate cells. Slides containing cell squashes were immediately immersed in liquid nitrogen and coverslips removed. Squashes containing isolated spermatocytes were used to identify by immunochemistry activated caspase-9, Rad51, $\gamma \mathrm{H} 2 \mathrm{AX}$, SYCP1 and SYCP3. Immunochemistry was as described above. As primary antibodies were used: to Rad51, Rabbit polyclonal (Abcam 1837); to $\gamma \mathrm{H} 2 \mathrm{AX}$, mouse polyclonal anti-histone-phospho (Ser139) (Millipore JBW301); to SYCP1, rabbit polyclonal (Abcam 15087); to SYCP3, rabbit polyclonal antibody (Abcam 12452). As secondary antibodies were used: goat anti-mouse antibodies conjugated with FITC (Jackson Laboratory); goat anti-mouse conjugated to Alexa 350 (Jackson Laboratory); goat anti-mouse conjugated with Texas Red (Jackson Labs); goat anti-rabbit conjugated to FITC (Jackson Laboratory); goat antirabbit conjugated with Texas Red (Jackson Laboratory).

Images were acquired using a Nikon (Tokyo,
Japan) Optiphot or an Olympus BX61 epifluorescent microscopes equipped with Nikon PL APO 100X, 1.30 NA objective lenses. Corresponding fluorescent signals were detected using the following barrier filters Chroma 49002 ET-GFP (FITC/Cy2), 49010 ET-RandB Phycoerythrin/mOrange/Mko and Hoechst UV2A. Images were acquired using a DS-L1 Nikon camera control unit. All images were minimally processed for contrast using Adobe Photoshop CS5.1 software.

\section{Statistic analysis}

Fisher's test was used for dichotomous variables. Significant differences $\mathrm{P}<0.05$ with a confidence interval of $95 \%$ were considered. The statistical analysis program Graph Pad 5.1 was used.

\section{Results}

\section{A brief and mild temperature increase induced apoptosis in mouse germ cells}

In untreated mice, we found 1 apoptotic spermatocyte, or that was positive for activated caspase-3 in situ, among of 1043 germ cells examined. Moreover, in untreated animals, Sertoli cells and round and elongated spermatids showed a very low frequency of apoptotic signals. These observations were consistent with a previous report. ${ }^{28}$ In contrast, mice subjected to a transient increase in scrotal temperature showed a significant augment in apoptotic spermatocytes. Immunocytochemistry revealed that activated caspase- 3 was found in $15 \%$ and $22 \%$ of spermatocytes after $4 \mathrm{~h}$ and $5 \mathrm{~h}$ post temperature exposure, respectively. Higher rates of apoptosis were detected $5 \mathrm{~h}$ elapsed after the end of treatment using antibody probes for activated caspase- 9 (Table 1). Since a higher enrichment for apoptotic spermatocytes (i.e., showing activated caspase-9 in situ) was observed at $5 \mathrm{~h}$ post higher scrotal temperature treatment (37\%) and cells also displayed wellpreserved nuclear morphology, apoptotic cell enrichment was conducted using these conditions for the studies that followed.

\section{Apoptotic spermatocytes were not randomly distributed during gametogenesis}

At the resolution of the light microscope, histological sections of seminiferous tubules obtained from specimens subjected to a higher transient scrotal temperature showed no abnormalities in cellular organization and cell associations at the different stages of gametogenesis. Suggesting therefore, that transient higher scrotal temperature conditions chosen for this 
study, had little or no effect on the integrity of seminiferous tubules. Examination of apoptotic spermatocyte populations revealed that they were not randomly distributed but instead, only observed in association with a few stages of the spermatogenic cycle. Most apoptotic spermatocytes were concentrated in stages III-IV and XIXII of the spermatogenic cycle. Stages III-IV and XI-XII showed spermatocytes at the middle of the pachytene stage and at metaphase I respectively (Figure 1). Of 1,043 apoptotic spermatocytes examined, $50 \%$ were in stages III-IV, about $44 \%$ were in stages XI-XII and about $4 \%$ were in stages IX-X of the spermatogenic cycle (Figure $2)$. In stages of the spermatogenic cycle, other than stages III-IV, XI-XII and IX-X, apoptotic spermatocytes were not observed. Similar results were obtained using immunofluorescent or peroxidase-based histochemical localizations (Figure 1).

\section{Identification of meiotic stages in isolated apoptotic spermatocytes}

Isolated spermatocytes and other gonial cells obtained from untreated mice and mice subjected to a transient increase in scrotal temperature, were morphologically indistinguishable at the level of resolution of the light microscope. In situ localization analyses, how- ever, revealed that apoptotic markers were preferentially abundant in spermatocytes and round spermatids. Furthermore, apoptotic spermatocytes were mainly in pachytene and at metaphase I stages. Specifically, of 869 apoptotic spermatocytes studied, $48 \%$ were in middle pachytene and $44 \%$ were in metaphase I. In contrast, about $6 \%$ of apoptotic spermatocytes were in diplotene. Moreover, apoptosis

Table 1. Activated caspases 3 and 9 in mouse spermatocytes after 4 or 5 h post 15 min exposed to a moderate rise of temperature.

\begin{tabular}{|c|c|c|c|c|}
\hline \multicolumn{5}{|c|}{ Spermatocytes exposed for $\mathbf{1 5} \mathrm{min}$ to a moderate rise in temperature } \\
\hline \multirow[t]{2}{*}{ Post temp expos. time } & \multicolumn{2}{|c|}{ Caspase 3: in situ activity } & \multicolumn{2}{|c|}{ Caspase 9: in situ activity } \\
\hline & Active & Inactive & Active & Inactive \\
\hline $4 \mathrm{~h}$ & $45(15 \%)$ & $265(86 \%)$ & $157(27 \%)$ & $427(72 \%)$ \\
\hline $5 \mathrm{~h}$ & $184(22 \%)$ & $671(78 \%)$ & $532(37 \%)$ & $912(63 \%)$ \\
\hline
\end{tabular}
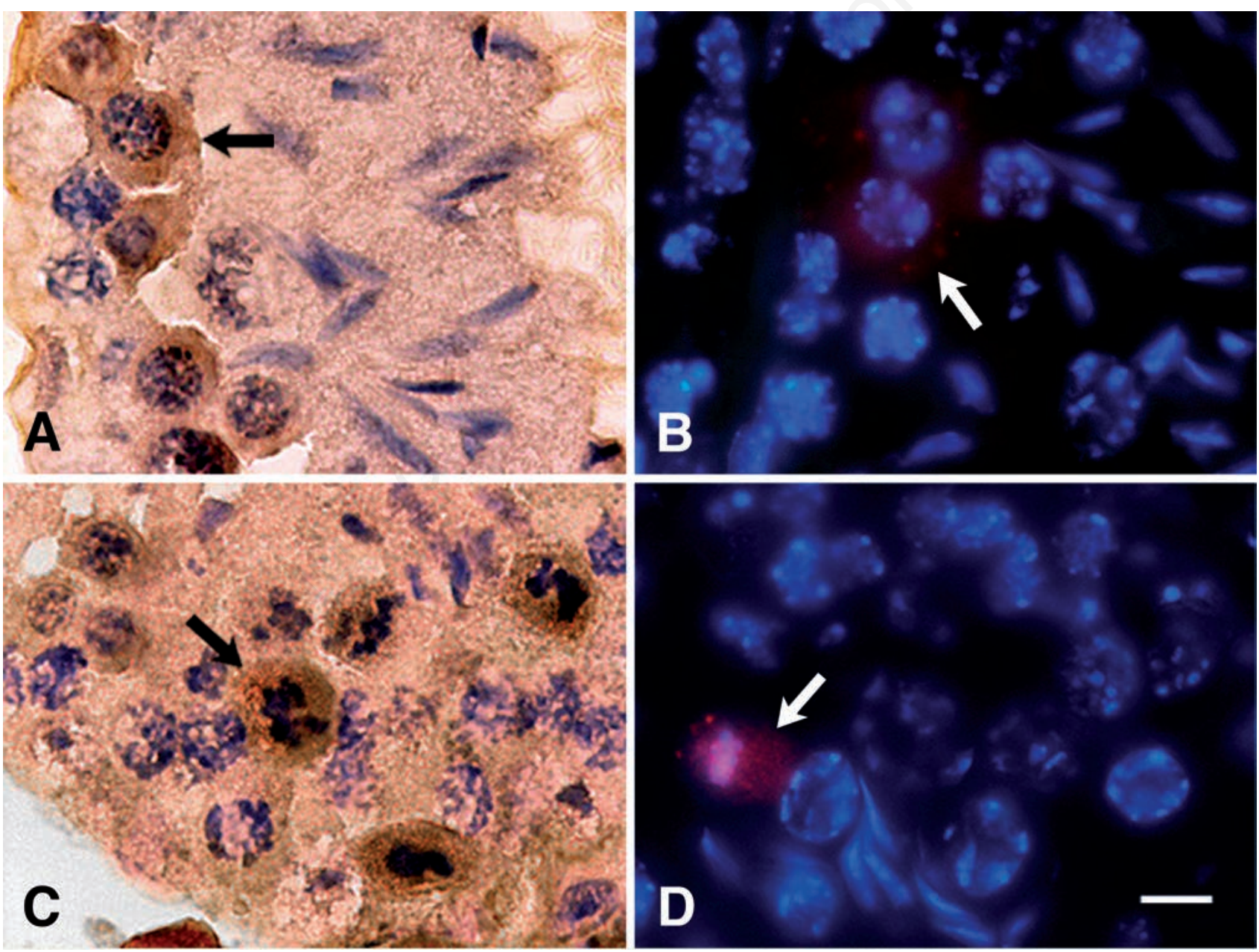

Figure 1. Immunocytochemical localization of activated caspase-9 to apoptotic spermatocytes in histological sections through seminiferous tubules from mice Mus m. domesticus. A-D) Independent fields. A,C) Bright field microscopy: immunoperoxidase-conjugated secondary antibodies and diaminobencidine (brown) detection of caspase-9 activation. B,D) Fluorescent microscopy: immunofluorescent detection of caspase-9 activation with Texas red-conjugated secondary antibodies (red). A,B) Apoptotic spermatocytes at pachytene in stages III-IV of the spermatogenic cycle; arrow points to an apoptotic spermatocyte at pachytene. C,D) Apoptotic spermatocytes at metaphase in stages XI-XII of the spermatogenic cycle; Arrow points to an apoptotic spermatocyte at metaphase I. Nuclei and DNA/chromatin were stained with Mayer's hematoxyline $(A, C)$ and DAPI $(B, D)$, respectively. Scale bars: $20 \mu \mathrm{m}$. 
markers were practically undetectable during leptotene and zygotene (Table 2, Figure 3). It should also be noted that sets of round spermatids, often showed positive reaction to activated caspases in their shared cytoplasm.

\section{Apoptotic spermatocytes contain a} relative high frequency of abnormal

\section{synapses}

Apoptotic spermatocytes showed relative high frequencies of abnormal synapses including, partial unsynapses, discontinuous chromosomal axes, splitting of chromosomal axes as well as irregular deposit of SYCP1 and SYCP3 on SCs (Figure 4 A,A'). Apoptotic sper-

Table 2. Distribution of meiotic stages in apoptotic spermatocytes.

\begin{tabular}{lc} 
Meiotic stages & Apoptotic spermatocytes \\
Prophase I & $489(56 \%)$ \\
Leptotene & $0(0 \%)$ \\
\hline Zygotene & $2(0.3 \%)$ \\
Early pachytene & $5(0.6 \%)$ \\
\hline Middle pachytene & $415(48 \%)$ \\
Late pachytene & $18(2 . \%)$ \\
\hline Diplotene & $49(6 \%)$ \\
Metaphase I & $380(44 \%)$ \\
Total & $869(100 \%)$ \\
\hline
\end{tabular}

matocytes may present one or more of these anomalies affecting one or several bivalents (Figure 4A). Of 754 apoptotic spermatocytes examined, $64 \%$ showed abnormalities on chromosomal axes, evidenced by abnormal distribution of SYCP3. Nuclear alterations involving SYCP3 were not observed in normal or nonapoptotic spermatocytes (Table 3). Frequency differences in nuclear anomalies between non-apoptotic and apoptotic spermatocytes, as evidenced by SYCP3 distribution, were highly significant $(\mathrm{P}<0.0001)$. Of 441 spermatocytes examined, $79 \%$ showed structural abnormalities on SCs as evidenced by SYCP1 distribution. In contrast, only $0.7 \%$ of normal or nonapoptotic spermatocytes showed abnormalities in chromosomal synapses (Table 3). Frequency differences in nuclear abnormalities between normal or non-apoptotic and apoptotic spermatocytes, as evidenced by SYCP1, were highly significant $(\mathrm{P}<0.0001)$.

\section{Distribution of chromatin and DNA} repair proteins in apoptotic spermatocytes

\section{$\gamma \mathrm{H} 2 \mathrm{AX}$}

During pachytene, in normal or non-apoptotic spermatocytes, $\gamma \mathrm{H} 2 \mathrm{AX}$ was only localized to XY bivalent's chromatin displaying a roughly elliptical shape attached to the inner face of the nuclear envelope. In sharp contrast to nor-

Table 3. Synapses status in apoptotic and non-apoptotic pachytene spermatocytes.

\begin{tabular}{lccc} 
Caspase 9: in situ activity & Marker & $\begin{array}{c}\text { Synapses/chromosomal axes } \\
\text { Normal }\end{array}$ & Abnormal \\
Active & & $93(21 \%)$ & $348(79 \%)$ \\
Inactive & SYCP1 & $147(99 \%)$ & $1(0.7 \%)$ \\
Active & & $275(37 \%)$ & $479(64 \%)$ \\
Inactive & SCYP3 & $417(100 \%)$ & $0(0 \%)$ \\
\hline
\end{tabular}

Table 4. Distribution of $\gamma \mathrm{H} 2 \mathrm{AX}$ and RAD51 in apoptotic and non-apoptotic pachytene spermatocytes.

\begin{tabular}{lccc} 
Caspase 9: in situ activity & Marker & \multicolumn{2}{c}{ Marker: in situ distribution } \\
& & Normal & Abnormal \\
Active & $\gamma$ H2AX & $273(36 \%)$ & $481(64 \%)$ \\
Inactive & & $417(100 \%)$ & $1(0.2 \%)$ \\
Active & RAD51 & $132(29 \%)$ & $328(71 \%)$ \\
Inactive & & $285(99 \%)$ & $3(1 \%)$ \\
\hline
\end{tabular}

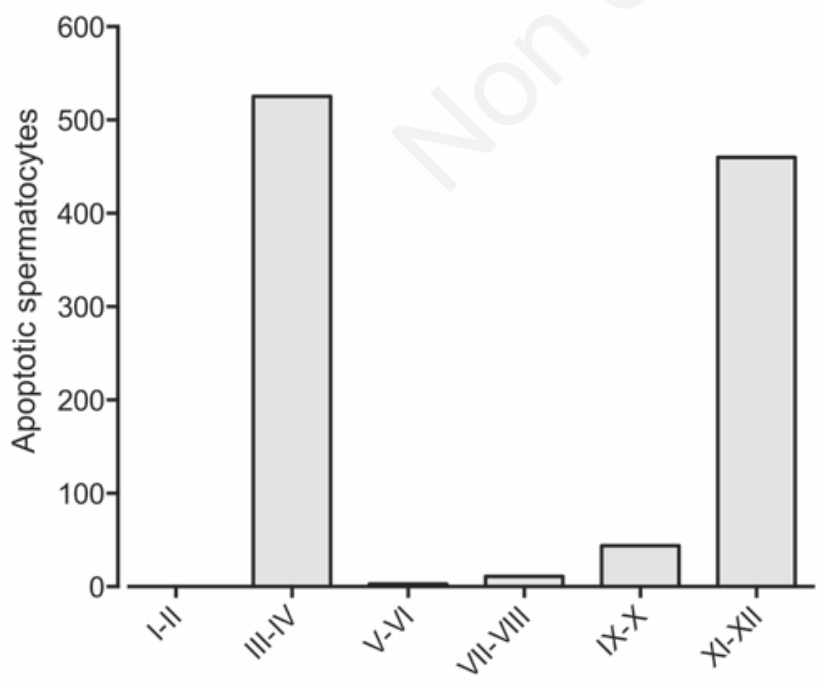

Figure 2. Relative distribution of apoptotic spermatocytes throughout the spermatogenic cycle. Bar graph showing the number of caspase-9 positive spermatocytes in each stage of the spermatogenic cycle of seminiferous tubules from heat stressed mice.

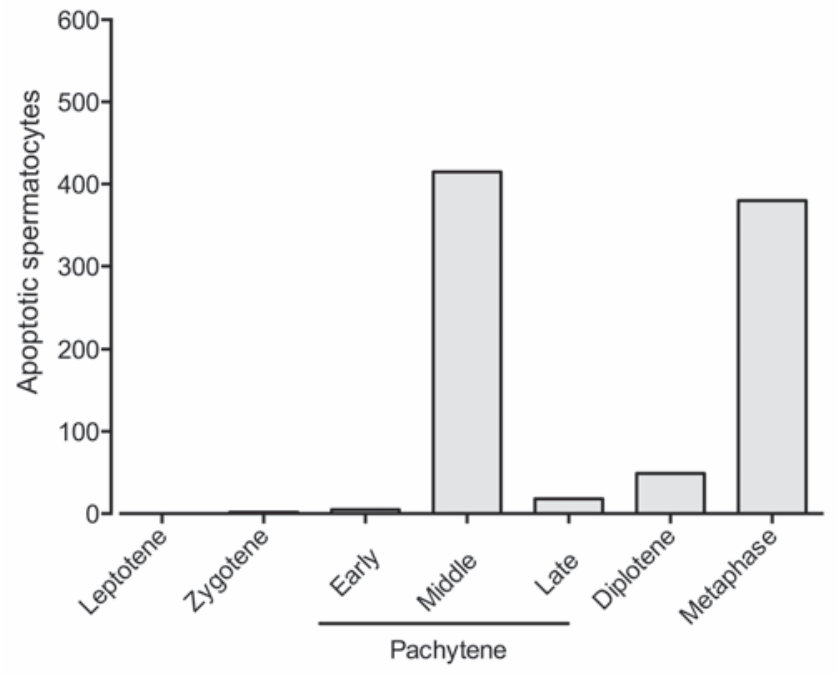

Figure 3. Relative distribution of apoptotic spermatocytes throughout prophase I and metaphase I. Bar graph showing the number of caspase-9 positive spermatocytes classified according to the different meiotic stages of isolated germ cells from heat stressed mice. 
mal spermatocytes, $\gamma \mathrm{H} 2 \mathrm{AX}$ remained associated with autosomal chromatin or chromosomal axes showing incomplete synapsis in apoptotic spermatocytes at pachytene. This association persisted throughout and was even observed in more advanced meiotic states, including late pachytene and diplotene. Moreover, in apoptotic spermatocytes at pachytene, $\gamma \mathrm{H} 2 \mathrm{AX}$ exhibited an atypical distribution over the sex chromosomes. Here $\gamma \mathrm{H} 2 \mathrm{AX}$ adopted a mainly ring shape tracing the axes of sex chromosomes but not extending to the central space occupied by the sex chromatin (not shown). Of 754 apoptotic spermatocytes in middle and late pachytene studied, $64 \%$ presented anomalous persistence of $\gamma \mathrm{H} 2 \mathrm{AX}$ over unsynapsed chromatin, as well as atypical distribution over sex chromosomes. Frequency differences in abnormal distribution of $\gamma \mathrm{H} 2 \mathrm{AX}$ between normal or non-apoptotic and apoptotic spermatocytes were highly significant $(\mathrm{P}<0.001)$ (Table 4).

\section{Rad51}

In normal or non-apoptotic spermatocytes, Rad51 was widely distributed throughout the chromatin at leptotene and over chromosomal axes at zygotene. Immunocytochemical detection of Rad51 decreased in tandem with the progression of chromosomal synapses. At pachytene, Rad51 was only detected in association with sex chromosomes' single axes (Figure 4B). In apoptotic spermatocytes at pachytene, Rad51 was observed in several foci over the path of autosomal bivalent SCs (Figure 4C). This unusual Rad51 distribution was also observed in apoptotic spermatocytes in late pachytene. Of 460 pachytene apoptotic spermatocytes studied, $71 \%$ showed persisting association of Rad51 with chromosomes. In these cells, detailed examination revealed that Rad51 formed several foci over autosomal bivalent's SCs. In 99\% of non-apoptotic spermatocytes, Rad51 was found associated with only the axes of sex chromosomes. Only in about $1 \%$ of these spermatocytes, Rad51 formed foci in autosomal bivalents (Table 4). Frequency differences of abnormalities between nonapoptotic and apoptotic spermatocytes were highly significant $(\mathrm{P}<0.001)$.

\section{Apoptotic spermatocytes showed abnormalities both in chromosome structure and DNA repair}

Apoptotic spermatocytes were also evaluated for the co-localization of structural proteins in chromosome axes and DNA repair proteins by examining the distribution of protein pairs SYCP3/ $\gamma$ H2AX and SYCP3/Rad51, respectively. Of 754 apoptotic spermatocytes examined, 48\% showed anomalies in the distribution of both synaptic and repair proteins.Specifically, $\gamma \mathrm{H} 2 \mathrm{AX}$ was found associated with chromatin surrounding discontinuous chromosomal axes as revealed by SYCP3 localization. In this population of apoptotic spermatocytes about 15\% showed anomalies in either synaptic or DNA repair proteins. About $20 \%$ of apoptotic spermatocytes showed no detectable alterations in the distribution of SYCP3 and $\gamma \mathrm{H} 2 \mathrm{AX}$ (Table 5). Of 460 apoptotic spermatocytes examined, approximately $51 \%$ showed anomalies in the distribution of both synaptic and DNA repair proteins. Rad51 was found in association with advanced pachytene stages and localized over chromosomal axes that appeared fractured. In this same population of apoptotic spermatocytes, only about $16 \%$ showed anomalies in chromosomal axes (as revealed by SYCP3 distribution) and about 20\% displayed abnormal Rad51 distribution. About $13 \%$ of apoptotic spermatocytes showed no alterations in the distribution of either of these proteins (Table 5).

Table 5. Co-distribution of SYCP3/ $\gamma \mathrm{H} 2 \mathrm{AX}$ and SYCP3/RAD51 in apoptotic pachytene spermatocytes.

\begin{tabular}{|c|c|c|c|c|}
\hline \multirow{3}{*}{ SYCP3 } & \multicolumn{3}{|c|}{ In situ distribution } & \multirow{2}{*}{ RAD51 } \\
\hline & & & & \\
\hline & Normal & Abnormal & Normal & Abnormal \\
\hline Normal & $156(21 \%)$ & $119(16 \%)$ & $62(14 \%)$ & $94(20 \%)$ \\
\hline Abnormal & $117(16 \%)$ & $362(48 \%)$ & $70(15 \%)$ & $234(51 \%)$ \\
\hline
\end{tabular}
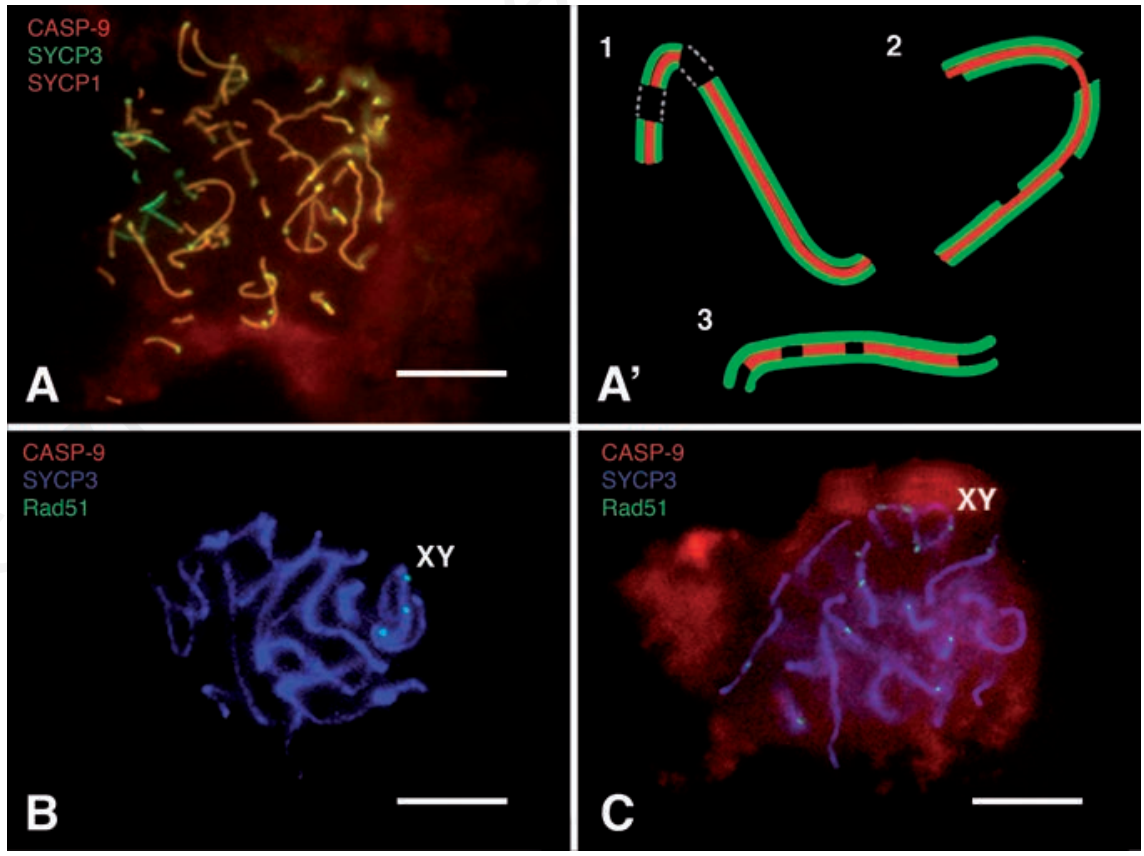

Figure 4. Immunofluorescent localization of activated caspase-9 and nuclear proteins SYCP1, SYCP3 and Rad51 in apoptotic and non-apoptotic spermatocytes at pachytene. A) In situ localization of SYCP1 (red) and SYCP3 (green) in an apoptotic pachytene spermatocyte evidenced by the wide reaction to activated caspase-9 (red) at the cytoplasm; several alterations in chromosome structure and synapses are detected in the nucleus; normal chromosome synapses are shown in yellow by overlaying fluorescent signals from SYCP1 (red) and SYCP3 (green). A') Apoptotic spermatocytes were isolated and probed with specific antibodies directed against activated caspase-9, SYCP1, SYCP3 and Rad51 as described in Materials and Methods; the panel shows alterations observed in SCs from apoptotic spermatocytes at pachytene: (1) Discontinuous chromosomal axes, (2) partial unsynapses, and (3) irregular distribution on SCs of SYCP1 or SYCP3. B) In situ localization of Rad51 (green) and SYCP3 (blue) in a normal pachytene spermatocyte; Rad51 is only detected associated to the sex chromosome single axes (XY). C) In situ localization of Rad51 (green) and SYCP3 (blue) in an apoptotic pachytene spermatocyte evidenced by the widespread cytoplasmic reaction to activated caspase-9 (red); Rad51 observed forming several foci (green) over the synaptonemal complexes of the autosomal bivalents (blue) in sex chromosome single axes (XY). Scale bars: $10 \mu \mathrm{m}$. 


\section{Discussion}

In the male germinal epithelium cellular responses leading to apoptosis normally occur at a very low frequency. This observation has limited our ability to study nuclear participants involved in triggering programmed germinal cell death. Previous studies by others have shown that a temporary rise in the temperature of the scrotum causes a complex response in germ cells including apoptosis. ${ }^{20,21,23,29}$ This temperature-precipitated response also involves an induction of genes associated with hypoxia and oxidative stress resulting in DNA damage and DSB. ${ }^{30,31}$ Moreover, mouse spermatocytes at pachytene experience higher oxidative stress responses compared to round spermatids when these stages are temporarily subjected to a temperature of $40^{\circ} \mathrm{C} .32$ The observation that spermatocytes have higher temperature sensitivity with respect to other germ cells offers an experimental approach for enrichment in apoptotic cells. Therefore, in this work we increased the number of germ cells undergoing apoptosis by exposing the scrotum of mice and consequently their seminiferous epithelia to a brief and moderate rise in temperature. ${ }^{23}$ Since among caspases, activation of caspase- 9 is considered a reliable marker for the initiation of the apoptotic response, ${ }^{33}$ in situ detection of activated caspase- 9 was used as a marker for evidencing initiation of apoptosis. Similarly as described in a rat model, ${ }^{23}$ a significant increase in germ cell apoptosis was obtained after $15 \mathrm{~min}$ of exposure to a mild scrotal temperature rise. This relative short exposure time followed by a $5 \mathrm{~h}$ delay in collecting specimens, lead to enrichment in apoptotic germ cells particularly in spermatocytes at pachytene and at metaphase I. Cell renewal dynamics in seminiferous epithelia, together with the relative short exposure time used in this study contributed to enriching for meiotic stages undergoing early apoptotic activation and reducing the chances of evaluating chromatin fragmentation and karyorrhexis, recognized as later events in apoptosis. ${ }^{2}$ Since apoptotic signals were predominantly found associated with spermatocytes at pachytene and at metaphase I, our results suggest that in seminiferous epithelia the apoptotic response specifically targets certain cell stages. Notably, these meiotic stages in turn coincide well with the onset of the pachytene and metaphase checkpoints. . $^{18,34-37}$ Observations in seminiferous epithelia also revealed that apoptotic spermatocytes were grouped in sets. Observation that support the hypothesis that cellular associations and intercellular bridges, that normally synchronize seminiferous epithelia development, may also be contributing to synchronize the triggering of apoptosis in these sets. ${ }^{38}$

Examining the spermatogenetic cycle, apoptotic spermatocytes concentrated in stages IIIIV, mostly at pachytene, IX-XII, mostly at metaphase I and, in IX-X mostly at advanced pachytene or diplotene. Our observations using isolated spermatocytes were consistent with those made while these cells still remained forming part of the seminiferous epithelium. ${ }^{39}$ Most isolated apoptotic spermatocytes were distributed between pachytene and metaphase I. An observation that is also consistent with the prophase and metaphase checkpoints. ${ }^{34-36}$ The prophase checkpoint ensures the correct recombination and synapsis of homologous chromosomes, ${ }^{34,35}$ while the metaphase checkpoint ensures the correct alignment and separation of homologous chromosomes; a process similar to what occurs during mitosis. ${ }^{36,37}$

To explain the small population of diplotene apoptotic spermatocytes we found at diplotene, two hypotheses have been put forward: That a prophase checkpoint evasion may be occurring through a mechanism known as MSUC (Meiotic Silencing of Unsynapsed Chromatin $)^{40}$ or, that there is a damage threshold requirement precipitating apoptosis. ${ }^{41}$ Since in this study the total time before specimens were prepared for immunocytochemistry was $5-1 / 4 \mathrm{~h}(15 \mathrm{~min}+5 \mathrm{~h})$ and the pachytene stage extends for 6 days, ${ }^{18}$ there would not be enough time for cells in early meiotic prophase to reach the diplotene stage. Therefore, we have to conclude, that most apoptotic spermatocytes at diplotene initiated apoptosis while at this stage. We do not have evidence however, to ascertain wether this late prophasic apoptosis involves the action of prophase or metaphase checkpoints. On the other hand, the absence of apoptosis between its highest peak during early prophase and a second peak toward the end of prophase does not support the hypothesis for sustained retention of the initial checkpoint. Therefore, the small population of apoptotic spermatocytes we found at diplotene, cannot be explained by the above hypotheses.

Our results show that there is indeed an association between spermatocyte apoptosis and abnormal synapses between homologous chromosomes. Synaptic anomalies were evident through changes in structural components of SCs. In this study, the quality of chromosomal axes or lateral elements of SCs were evaluated by examining the distribution SYCP3. The continuity of the SCs' central element was evaluated by examining the distribution of SYCP1. Although SYCP1 and SYCP3 are not the only components of SCs, they have been recognized as reliable in situ targets for evaluating synapses because they are highly conserved and widely distributed in SCs from several organisms. ${ }^{6,8}$ Indeed, assembly of SC components is a complex process regulated by a combination of associated nonstructural proteins and post-translational modifications, which together coordinate the timing of homologous chromosome pairing, DSB formation and recombination. ${ }^{42}$

Therefore, the heat stress may be directly affecting the distribution of synaptic proteins like SYCP3 or SYCP1, or it may be affecting others related to or interacting with $\mathrm{SC}$ assembly, like modified histones, DNA repair proteins, recombinases, etc. All of them are required for the normal synapsis and maintenance of the SC and consequently for the normal progression of the meiotic prophase. ${ }^{43}$ While conducting these in situ studies, we noticed that in apoptotic spermatocytes at pachytene, a phosphorylated form of H2AX persisted in association with chromatin and so did Rad51 in chromosomal axes. Both observations point to a delayed localization of these proteins with respect to their normal distribution pattern in meiotic prophase. Recruitment and renewal of these proteins is controlled by the meiotic prophase checkpoint. ${ }^{44}$ Pachytene spermatocytes carrying a minimum threshold of anomalies in protein recruitment, as we observed, would be detected by the meiotic prophase checkpoint and therefore eliminated by apoptosis. It is possible that these early meiotic events (i.e., synapsis, recombination) may be especially susceptible to a scrotal temperature rise, in similar way to oxidative stress. ${ }^{31,45}$ The persistence of $\gamma \mathrm{H} 2 \mathrm{AX}$ and Rad51 proteins through pachytene suggests that MSUC may indeed be occurring. If this is the case, repression of gene expression may also be occurring. ${ }^{40,46,47}$

Since the formation of synapses and DNA repair are intimately ligated, it is not possible to infer whether meiotic checkpoints act independently detecting alterations on synapses or on DNA repair. ${ }^{48}$ Moreover, our study could not discern sub-microscopical or molecular alterations occurring concurrently with or preceding checkpoints activation. Unfortunately, little is known about the role of checkpoints on meiotic divisions. Information governing meiotic anaphase chromosome separation is apparently contained within chromosomes and, does not involve the spindle. ${ }^{26,49}$ Checkpoint mechanisms detecting alterations in the two distinct meiotic divisions may also be an intrinsic property of meiotic chromosomes. However, many protein families has been described involved in chromatid cohesion or in the centromeric region organization, all of which may be subject to the metaphase checkpoint control. It has been described recently that some proteins first localize to the SCs' central regions subsequently they re-localize to the centromeric region of metaphasic chromo- 
somes. ${ }^{50}$ Thus, this complex process of chromosome segregation appears to be not only dependent on chromosome structure or on the integrity of the meiotic spindle, but on the coordination of multiple events including some whose dynamics starts at SCs of the preceding prophase I. ${ }^{50}$

We found that almost half of apoptotic spermatocytes were in metaphase I. Considering the time frame of our experiments, it would not be possible for damaged chromosomes at prophase to reach metaphase. Thus it is likely that the rise in scrotal temperatures induce damage during metaphases's chromosome alignment, or during the meiotic spindle assembly. Anomalies in each or all these steps would have been detected by the metaphase I checkpoint, which plays an important role in male germ cell development and regulation. ${ }^{51}$

This study showed evidence that anomalies in chromosome synapses and DNA repair, were frequently found in apoptotic spermatocytes at specific stages of the seminiferous epithelia. In our experiments, all gonadic cells were exposed moderate rise in temperature, but only a few specific germ cells were irreversibly damaged and underwent apoptosis. Since apoptotic spermatocytes were at an early prophase stage, at a stage when critical events are occurring under the surveillance of checkpoints capable of triggering apoptosis, it is possible to conclude that the enrichment for apoptotic spermatocytes observed was due to the induction of chromosomal alterations and the consequent activation of these checkpoints. These observations help explain the fertility decline observed in scrotal individuals who have had the temperature of their gonads elevated above their normal value.

\section{References}

1. Nakanishi Y, Shiratsuchi A. Phagocytic removal of apoptotic spermatogenic cells by Sertoli cells: mechanisms and consequences. Biol Pharm Bull 2004; 27:13-6.

2. Koji T, Hishikawa Y. Germ cell apoptosis and its molecular trigger in mouse testes. Arch Histol Cytol 2003; 66:1-16.

3. Codelia V, Cisternas P, Moreno R. Relevance of caspase activity during apoptosis in pubertal rat spermatogenesis. Mol Reprod Develop 2008;75:881-9.

4. Moens PB, Spyropoulos B. Immunocytology of chiasmata and chromosomal disjunction at mouse meiosis. Chromosoma 1995; 104: 175-182.

5. Mahadevaiah SK, Turner JM, Baudat F, Rogakou EP, de Boer P, Blanco-Rodriguez $\mathrm{J}$, et al. Recombinational DNA doublestrand breaks in mice precede synapsis.
Nat Genet 2001;27:271-6.

6. Zickler D, Kleckner N. Recombination, pairing, and synapsis of homologs during meiosis. Cold Spring Harb Perspect Biol 2015;7.pii:a016626.

7. Fraune J, Schramm S, Alsheimer M, Benavente R. The mammalian synaptonemal complex: protein components, assembly and role in meiotic recombination. Exp Cell Res 2012;318:1340-6.

8. Heyting C. Synaptonemal complexes: structure and function. Curr Opin Cell Biol 1996;8:389-96.

9. Yang F, Wang PJ. The Mammalian synaptonemal complex: a scaffold and beyond. Genome Dyn 2009;5:69-80.

10. Baudat F, Imai Y, de Massy B. Meiotic recombination in mammals: localization and regulation. Nat Rev Genet 2013;14:794-806.

11. Hunter N. Meiotic Recombination. In: A. Aguilera and R. Rothstein (eds.) Topics in current genetics. Molecular genetics of recombination. Springer-Verlag, Heidelberg, 2006; pp. 381-442.

12. Xiao A, Li H, Shechter D, Hee AS, Fabricio LA, Erdjument-Bromage $\mathrm{H}$, et al. WSTF regulates the H2A.X DNA damage response via a novel tyrosine kinase activity. Nature 2009;457:57-62.

13. Cook PJ, Ju BG, Telese F, Wang X, Glass CK, Rosenfeld MG. Tyrosine dephosphorylation of H2A.X modulates apoptosis and survival decisions. Nature 2009;458:591-6.

14. Fernández-Capetillo 0, Mahadevaiah SK, Celeste A, Romanienko PJ, CameriniOtero RD, Bonner WM, et al. H2AX is required for chromatin remodeling and inactivation of sex chromosomes in male mouse meiosis. Dev Cell 2003;4:497-508.

15. Ichijima Y, Sin HS, Namekawa SH. Sex chromosome inactivation in germ cells: emerging roles of DNA damage response pathways. Cell Mol Life Sci 2012;69:2559-72.

16. Neale MJ, Keeney S. Clarifying the mechanics of DNA strand exchange in meiotic recombination. Nature 2006; 442:153-8.

17. Lao JP, Cloud V, Huang CC, Grubb J, Thacker D, Lee CY, et al. Meiotic crossover control by concerted action of Rad51-Dmc1 in homolog template bias and robust homeostatic regulation. PLoS Genet 2013;9: e1003978.

18. Cohen PE, Pollack SE, Pollard JW. Genetic analysis of chromosome pairing, recombination, and cell cycle control during first meiotic prophase in mammals. Endocrine Rev 2006;27:398-426.

19. Moens PB, Marcon E, Shore JS, Kochakpour N, Spyropoulos B. Initiation and resolution of interhomolog connections: crossover and non-crossover sites along mouse synaptonemal complexes. J Cell Sci 2007;120:1017-27.

20. Rockett JC, Mapp FL, Garges JB, Luft JC, Mori C, Dix DJ. Effects of hyperthermia on spermatogenesis, apoptosis, gene expression, and fertility in adult male mice. Biol Reprod 2001;65:229-39.

21. Yaeram J, Setchell BP, Maddocks S. Effect of heat stress on the fertility of male mice in vivo and in vitro. Reprod Fertil Dev 2006;18:647-53

22. Banks S, King SA, Irvine DS, Saunders PTK. Impact of a mild scrotal heat stress on DNA integrity in murine spermatozoa. Reproduction 2005:129:505-14.

23. Sinha Hikim AP, Lue Y, Yamamoto C, Vera Y, Rodriguez S, Yen PH, et al. Key apoptotic pathways for heat-inducedprogrammed germ cell death in the testis. Endocrinology 2003;144:3167-75.

24 McManus JF. Histological demonstration of mucin after periodic acid. Nature 1946;158:202-14.

25. Oakberg EF. A description of spermiogenesis in the mouse and its use in analysis of the cycle of the seminiferous epithelium and germ cell renewal. Am J Anat 1956;99:391-413.

26. Eaker S, Pyle A, Cobb J, Handel MA. Evidence for meiotic spindle "checkpoint" from analysis of spermatocytes from Robertsonian-chromosome heterozygous mice. J Cell Sci 2011;114:2953-65.

27. Page J, Suja JA, Santos JL, Rufas JS. Protein inmunolocalization in meiotic cells by means a new squash procedure. Chromosome Res 1998;6:639-42.

28. Blanco-Rodríguez J. Keep cycling or died: the role of germ cell apoptosis in spermatogenesis. Recent Prog Horm Res 2002;57:75-101.

29. Lue Y, Sinha Hikim AP, Swerdloff RS, Im P, Taing KS, Bui T, et al. Single exposure to heat induces stage-specific germ cell apoptosis in rats: role of intratesticular testosterone on stage specificity. Endocrinology 1999;140:1709-17.

30. Paul C, Melton DW, Saunders PT. Do heat stress and deficits in DNA repair pathways have a negative impact on male fertility? Mol Hum Reprod 2008;14:1-8.

31. Paul C, Teng S, Saunders PTK. A single, mild, transient scrotal heat stress causes hypoxia and oxidative stress in mouse testes, which induces germ cell death. Biol Reprod 2009;80:913-9.

32. Pino JA, Osses N, Oyarzún D, Farías JG, Moreno RD, Reyes JG. Differential effects of temperature on reactive oxygen/nitrogen species production in rat Pachytene spermatocytes and round spermatids. Reproduction 2013;145:203-12.

33. Shi Y. Caspase activation, inhibition, and 
reactivation: a mechanistic view. Protein Sci 2004;13:1979-87.

34. Roeder GS, Bailis JM. The pachytene checkpoint. Trends Genet 2000;16:395-403.

35. Subramanian VV, Hochwagen A. The meiotic checkpoint network: Step-by-step through meiotic prophase. Cold Spring Harb Perspect Biol 2014;6:a016675

36. Hasthorpe S, Tainton K, Peart M, Roeszler KN, Bell KM, Lusby PE, et al. G2/M checkpoint gene expression in developing germ cells. Mol Reprod Dev 2007;74:531-8.

37. Xu YR, Dong HS, Yang WX. Regulators in the apoptotic pathway during spermatogenesis: killers or guards? Gene 2016;582:97-111.

38. de Rooij DG. Proliferation and differentiation of spermatogonial stem cells. Reproduction 2001;121:347-54.

39. Ashley T, Gaeth AP, Creemers LB, Hack AM, de Rooi DG. Correlation of meiotic events in testis sections and microspreads of mouse spermatocytes relative to the midPachytene checkpoint, Chromosoma 2004;113:126-36.

40. Burgoyne PS, Mahadevaiah SK, Turner JM. The consequences of asynapsis for mammalian meiosis. Nat Rev Genet 2009;
10:207-16.

41. Mahadevaiah SK, Bourc'his D, de Rooij DG, Bestor TH, Turner JM, Burgoyne PS. Extensive meiotic asynapsis in mice antagonises meiotic silencing of unsynapsed chromatin and consequently disrupts meiotic sex chromosome inactivation. J Cell Biol 2008;182:263-76.

42. Cahoon CK, Hawley RS. Regulating the construction and demolition of the synaptonemal complex. Nat Struct Mol Biol 2016;23:369-77.

43. Longhese MP, Bonetti D, Guerini I, Manfrini N, Clerici M. DNA double-strand breaks in meiosis: checking their formation, processing and repair. DNA Repair 2009;8:1127-38.

44. de Rooij DG, de Boer P. Specific arrests of spermatogenesis in genetically modified and mutant mice. Cytogenet Genome Res 2003;103:267-76.

45. Vera Y, Rodriguez, S, Castanares M, Lue Y, Ateinza V, Wang $\mathrm{C}$, et al. Functional role of caspases in heat-induced testicular germ cell apoptosis. Biol Reprod 2005;72:516-22.

46. Turner JM, Mahadevaiah SK, FernandezCapetillo 0, Nussenzweig A, Xu X, Deng
CX et al. Silencing of unsynapsed meiotic chromosomes in the mouse. Nat Genet 2005;37:41-7.

47. Baarends WM, Wassenaar E, van der Laan R, Hoogerbrugge JW, Sleddens-Linkels E, et al. Silencing of unpaired chromatin and histone H2A ubiquitination in mammalian meiosis. Mol Cell Biol 2005;25:1041-53.

48. Li X, Schimenti JC. Mouse pachytene checkpoint 2 (trip13) is required for completing meiotic recombination but not synapsis. PLos Genet 2007;3:e130.

49. Paliulis LV, Nicklas RB. The reduction of chromosome number in meiosis is determined by properties built into the chromosomes. J Cell Biol 2000;150:1223-31.

50. Gómez R, Felipe-Medina N, Ruiz-Torres M, Berenguer I, Viera A, Pérez $\mathrm{S}$, et al. Sororin loads to the synaptonemal complex central region independently of meiotic cohesin complexes. EMB0 Reports 2016;17:695-707.

51. Print CG, Loveland KL. Germ cell suicide: new insights into apoptosis during spermatogenesis. BioEssays 2000;22:423-30. 